Rev. Elev. Méd. vét. Pays trop., 1970, 23 (1) : 43-48

\title{
La cysticercose bovine dans la région de Fort-Lamy L'infestation naturelle des jeunes
}

\author{
par M. GRABER (*)
}

\begin{abstract}
RESUME
Chez des yeaux de lait sacrifués dans la région de Fort-Lamy, la cysticercose à Cysticercus bovis est relativement peu fréquente 3 p. 100).

L'infestation se produit à partir du sevrage. Entre 1 et 2 ans, le nombre d'animaux parasıtés atteint 31 p. 100 pour décroître par la suite.

Les conséquences de cet état de choses en matière de veaux de boucherie et de "baby-beef" sont envisagées.
\end{abstract}

\section{INTRODUCTION}

La cysticercose bovine Cysticercus bovis a déjà fait, au Tchad, l'objet de plusieurs éudes (GRABER, 1959; GRABER et THOME, 1964; GRABER et TABO, 1968) et l'on peut considérer que, dans ses grandes lignes, cette zoonose est actuellement assez bien connue.

Cependant, quelques points demeurent obscurs, notamment l'âge d'infestation des jeunes veaux.

Ce problème mérite de retenir l'attention, car, à l'abattoir de Fort-Lamy, les animaux de cette catégorie sont sacrifiés en nombre de plus en plus élevé chaque année. Pour éviter la stérilisation, toujours onéreuse, des carcasses ladres, on a intérêt à connaître l'époque exacte où la cysticercose fait son apparition, de manière à orienter, au départ, les achats vers du bétail non encore parasité.

Le présent travail résume une série d'observations faites au laboratoire de Farcha de 1965 à 1968.

(*) Institut d'Elevage et de Médecine Vétérinaire des Pays Tropicaux - Laboratoire de Farcha-FortLamy.

\section{MATERIEL ET METHODES}

$\mathrm{Au}$ total, 458 animaux originaires de la région de Fort-Lamy ont été utilisés de 1963 à 1968, à savoir :

- 66 veaux de Jait (groupe A),

- 263 bouvillons (groupe B),

- 129 vieux animaux (groupe C) $\left(^{*}\right.$ ).

La moyenne des poids, selon les années, oscillait entre 26,6 et 30,2 ktlogrammes pour le groupe A, 111 et 168 kilogrammes pour le groupe B et 257 et 280 kilogrammes pour le groupe $C$.

En milieu d'élevage traditionnel, il est particulièrement difficile de se procurer des veaux de lait que leurs propriétaires, pour diverses raisons, se refusent à vendre. Le prix demandé est toujours beaucoup plus élevé (1 fois et demi à 2 fois) que celui des animaux de la classe d'âge supérieure, ce qui limite considérablement les possibilités d'investigation.

Dans le cas présent, l'enquête n'a pu être effectuée qu'à la faveur d'essais thérapeutiques

(“) En général, des vieilles vaches de réforme appartenant à des éleveurs sédentaires. 
qui ont nécessité l'achat et l'autopsie d'un assez grand nombre de jeunes.

La technique mise en ouvre est simple : elle consiste à comparer les taux de cysticercose dans les groupes $\mathbf{A}, \mathrm{B}$ et $\mathrm{C}$ et, en fonction du poids, à définir l'âge le plus favorable à l'infestation par Cysticercus bovis.

Pour ce faire, les carcasses des animaux examinés sont découpées en menus morceaux et les cysticerques soigneusement recherchés dans les muscles et les organes. Is sont comptés et leur survie est appréciée dans de la bile de bouf placée dans une étuve maintenue à $39^{\circ} \mathrm{C}$.

\section{RESULTATS}

\section{Résultats globaux}

Ils figurent aux tableaux $\mathrm{n}^{\circ s} 1$ et 2 , ainsi qu'au graphique $\mathrm{n}^{0} 1$.

Jl apparaît nettement que la cysticercose des veaux de moins de 40 kilogrammes est, dans la région de Fort-Lamy, relativement peu fréquente, 3 p. 100 en moyenne. Les deux animaux atteints pesaient 27 à 32 kilogrammes et étaient âgés de 3 mois - 3 mois et demi environ. On sait qu'il faut en principe 10 à 12 semaines (*) après l'absorption d'oufs de de Taenia saginata pour obtenir la formation

TABLEAU $\mathrm{NI}^{\circ} \mathrm{I}$

Pourcentage d'animaux porteurs de Cysticerques.

\begin{tabular}{|c|c|c|c|c|c|c|}
\hline \multirow[b]{2}{*}{ Années } & \multicolumn{2}{|c|}{ Veeux de moins de $40 \mathrm{~kg}$} & \multicolumn{2}{|c|}{ Bouvillons } & \multicolumn{2}{|c|}{ Vieux animaux } \\
\hline & $\begin{array}{l}\text { Poids* } \\
\text { moyen } \\
\text { (en kg) }\end{array}$ & $\begin{array}{l}\text { Tombre d'animaux } \\
\text { parasités et } \\
\text { pourcentage }\end{array}$ & $\begin{array}{l}\text { Poids* } \\
\text { moyen } \\
\left.\text { (en } k_{g}\right)\end{array}$ & $\begin{array}{l}\text { Nambre a'enimeux } \\
\text { peresités et } \\
\text { pourcentage }\end{array}$ & $\begin{array}{l}\text { Poids } \\
\text { moyen } \\
\text { (en kg) }\end{array}$ & $\begin{array}{l}\text { Nombre d'animaux } \\
\text { parasités et } \\
\text { pourcentage }\end{array}$ \\
\hline $\begin{array}{l}1963 \\
1964\end{array}$ & - & - & 128 & $24 \operatorname{sur}_{(27,9)} 86$ & 267,1 & $\begin{array}{l}8 \operatorname{sur}_{2} 55 \\
(14,54)\end{array}$ \\
\hline 1965 & 27,9 & $0 \sin _{(0)} 14$ & 168 & $\begin{array}{c}7 \operatorname{sur} \\
(15,9)\end{array}$ & 272 & $\begin{array}{c}2 \operatorname{sur} 29 \\
(6,89)\end{array}$ \\
\hline 1966 & 26,6 & $0 \operatorname{sur}_{(0)} 24$ & III & $\begin{array}{r}5 \operatorname{sur} 24 \\
(20,83)\end{array}$ & 280 & $2 \operatorname{sur}_{(\theta)} 25$ \\
\hline $\begin{array}{l}1967 \\
1968\end{array}$ & 30,2 & $2 \operatorname{sur}_{(7,1)} 28$ & 143,2 & $\begin{array}{c}21 \text { sur } 109 \\
(19,26)\end{array}$ & 256,7 & $2 \operatorname{sur}_{(10)} 20$ \\
\hline motal & 29,2 & $2 \operatorname{sur}_{(3)} 66$ & 137,5 & $\begin{array}{c}57 \underset{\operatorname{sur}}{263} \\
(21,66)\end{array}$ & 266,4 & $\begin{array}{l}14 \operatorname{sur} 129 \\
(10,85)\end{array}$ \\
\hline
\end{tabular}

TABLEAU HO II

Cysticercose des jeunes zébus en fonction du poids et de l'âge.

\begin{tabular}{|l|c|c|c|c|}
\hline $\begin{array}{c}\text { Poids } \\
\text { (en kilogrammes) }\end{array}$ & $\begin{array}{c}\text { Age } \\
\text { approximatif }\end{array}$ & $\begin{array}{c}\text { Iombre d'animaux } \\
\text { examinés }\end{array}$ & $\begin{array}{c}\text { Nombre d'animaux } \\
\text { parasités }\end{array}$ & $\begin{array}{c}\text { Pourcentage } \\
\text { d'infestation }\end{array}$ \\
\hline $\begin{array}{l}\text { Veaux de leit } \\
\text { (moins de } 40 \mathrm{~kg} \text { ) }\end{array}$ & 1 jour à 5 mois & 66 & 2 & 3 \\
\hline $\begin{array}{l}\text { Bouvillons de } \\
40 \text { à } 100 \mathrm{~kg}\end{array}$ & 6 mois à 1 an & 45 & 11 & 24,4 \\
\hline $\begin{array}{l}\text { Bouvillons de } \\
\text { Loo à l60 kg }\end{array}$ & 1 an à 2 ans & 97 & 30 & 30,9 \\
\hline $\begin{array}{l}\text { Bouvillons de } \\
\text { l60à 240kg }\end{array}$ & 2 ans à 3 ans & 43 & 6 & 14 \\
\hline
\end{tabular}

(*) 18 semaines selon Mc MANUS. 


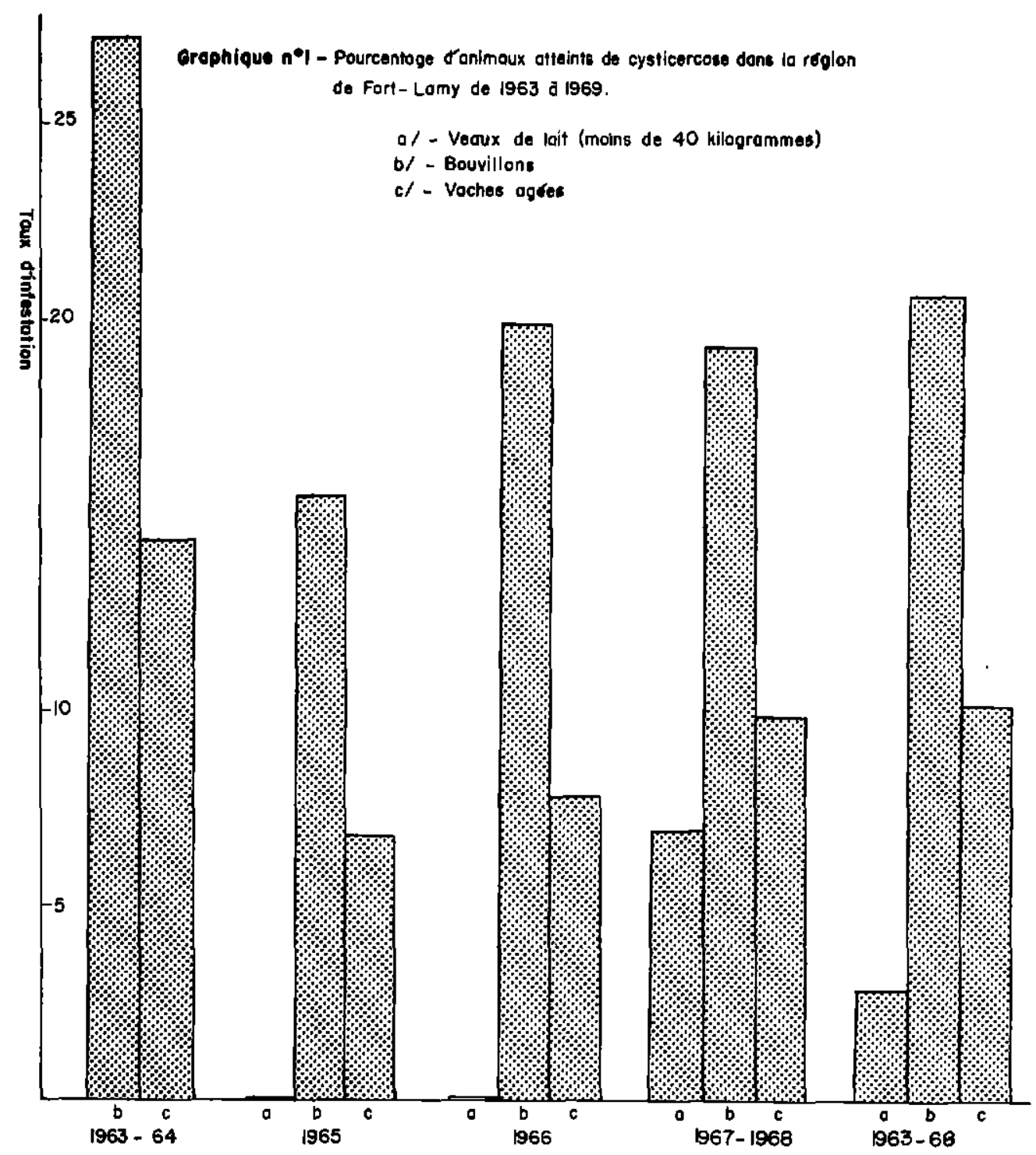

de cysticerques (Mc INTOSH et MILLER, 1960). Ce délai est susceptible de varier en fonction de divers facteurs, notamment la réaction inflammatoire de l'hôte (SILVERMAN et HULLAND, 1961) ou la localisation des vésicules, celles des masséters ou du myocarde se développant plus rapidement que les autres (VAN GILS, 1963).

Dans ces conditions, on peut considérer que le veau est capable de contracter la cysticercose dans les premiers jours qui suivent la naissance ou même, selon certains auteurs, avant celle-ci.

Ces faits sont bien connus et ont déjà été signalés dans l'Est africain, au Kenya notam- ment (URQUHART, 1961) où l'incidence chez les jeunes de 6 à 20 semaines semble beaucoup plus élevée (de l'ordre de 50 p. 100) qu'au Tchad.

Dans le groupe B (bouvillons de 40 à 240 kilogrammes) le pourcentage moyen de 1963 à 1969 est de 21, 66 p. 100 , à peine supérieur à ce qu'il était pour la période 19541962 (19,96 p. 100). Il y a donc 7 fois plus d'animaux ladres que dans la tranche d'âge précédente, ce qui donne déjà une première indication sur l'époque la plus favorable à l'infestation du zébu.

Dans le groupe $\mathrm{C}$ qui comprend des adultes de $9-10$ ans et plus, 11 p. 100 des vaches 
ẫgées sacrifiées sont atteintes. Dans 85 p. 100 des cas, les parasites sont vivants. Il s'agit soit d'une cysticercose ancienne à évolution très lente, ce qui est loin d'être la règle générale au Tchad, soit plutôt d'une cysticercose récente touchant des sujets neufs non encore immunisés (GRABER et THOMÉ, 1964) par une atteinte antérieure.

\section{Résultats en fonction du poids et de l'âge}

Arbitrairement, les 251 animaux ayant fait l'objet de la seconde partie de l'enquête ont été divisés en:

- veaux de lait de moins de 40 kilogrammes,

- bouvillons de 40 à 100 kilogrammes,

- bouvillons de 100 à 160 kilogrammes,

— bouvillons de 160 à 240 kilogrammes.

L'examen de la dentition a permis d'apprécier leur âge approximatif, bien qu'en cette matière, chez le zébu, les normes ne soient pas encore complètement fixées.

La lecture du tableau $\mathbf{n}^{\circ} 2$ montre que le nombre d'animaux porteurs de cysticerques augmente considérablement à partir de 6 mois, passe par un maximum entre un et deux ans et décroît sensiblement entre deux et trois ans, avec présence, dans ce cas, de très nombreux cysticerques calcifiés ou en passe de le devenir.

Compte tenu de ce qui a été dit dans le paragraphe précédent concernant le temps mis par un ouf de Taenia saginata pour donner chez le zébu une vésicule ladre, les plus fortes infestations semblent se produire à partir de 3 mois - 3 mois et demi, ce qui correspond au début de la période de sevrage. Au Tchad, en effet, les veaux de lait sont presque toujours parqués dans des enclos sommairement clôturés, bien visibles et situés dans le village même ou légèrement en dehors. Ils sont souvent attachés à des piquets ou groupés le long d'une corde. Les mères ne nourrissent leurs jeunes que le matin et le soir. Dans la journée, les parcs peu fréquentés sont laissés à la garde d'un berger, ce qui limite la dispersion fécale. $\mathrm{Au}$ bout d'un certain temps, variable selon les animaux, les régions, les populations (très tard au Mayo Kebbi) et les besoins en lait (surtout à la périphérie des villes), les veaux sont peu à peu sevrés et lachés en groupe, en dehors des zones habitées, tout en demeurant dans leur périmètre immédiat.

En l'absence quasi totale de lieux d'aisance, ils ont toute chance de rencontrer sur le sol, soit des anneaux de ténia humain émis récemment, soit des ceufs qui sont absorbés facilement, car, à certaines époques de soudure alimentaire particulièrement difficile (fin de la saison sèche en juin-juillet), la coprophagie est à peu près générale. Aussi, dans la région de Fort-Lamy, la cysticercose est-elle surtout une affection d'hivernage (GRABER et THOMÉ, 1964)

L'infestation diminue progressivement au fur et à mesure que les bouvillons sont incorporés au gros du troupeau qui va paître en brousse, loin d'éventuels agents disséminateurs.

En définitive, si, de par le mode d'élevage en vigueur au Tchad, les possibilités de contamination des veaux de lait semblent réduites, il n'en est plus de même à partir du sevrage où les conditions deviennent alors très favorables.

\section{CONSEQUENCES PRATIQUES}

Les conséquences pratiques de ces observations concernent les veaux de boucherie et le «baby-beef », depuis qu'au Tchad l'interdiction dabattage des jeunes a été levée.

On a intérêt bien entendu à choisir des animaux indemnes. Le diagnostic sérologique, du vivant de l'animal, permet théoriquement d'éliminer les porteurs de cysticerques. Malheureusement, ces méthodes, malgré de nombreuses recherches, ne sont actuellement pas très au point. Elles ne tiennent compte, en général, ni des polyparasitismes si fréquents en Afrique centrale, ni de la présence dans l'intestin d'un certain nombre de grands cestodes appartenant au groupe des Anoplocephalidae qui peuvent déterminer des fausses réactions positives. Cet inconvénient n'est pas négligeable car, autour de Fort-Lamy, 7 p. 100 des veaux de lait et 15 p. 100 des bouvillons (moyenne 1954-1969) hébergent Moniezia expansa, Moniezia benedeni ou Thysaniezia ovilla.

En attendant d'y voir plus clair, les veaux de boucherie sont achetés très jeunes avant le sevrage, isolés dans des conditions d'hygiène sévère et convenablement engraissés. 
Aussi, à l'abattoir de Fort-Lamy, sur les 11.535 «veaux » sacrifiés de 1958 à 1969 n'a-t-il été rencontré que 111 cas de cysticercose, soit 0,96 p. 100.

La différence entre le pourcentage de l'abattoir et celui donné par la section de parasitologie tient au fait que les conditions d'examen ne sont pas les mêmes: dans le premier cas, pour diverses raisons (résistance des bouchers; risques de contamination bactérienne), les épaules ne sont pas "levées » et il n'est pas non plus pratiqué d'incisions au niveau des muscles adducteurs de la cuisse. Or, au laboratoire, l'expérience prouve que ce sont des zones où, chez le veau, le parasite se localise électivement.

Quant au «baby-beef », il en a été fortement question pour alimenter certains marchés d'exportation. La chose est possible : des essais réalisés durant l'hivernage 1968 sur la concession du laboratoire ont montré que des bouvillons de 150 kilogrammes, dûment vaccinés et protégés contre les maladies parasitaires externes et internes étaient capables de prendre en moyenne 46 kilogrammes en 45 jours et 80 kilogrammes en 95 jours.

La nourriture fournie par le pâturage naturel a été complétée par des distributions modérées de graines de coton dans la première expérience, de paille sèche dans la seconde. La viande était d'excellente qualité.

Il est bien évident que, dans cette classe d'âge, la cysticercose qui touche entre 25 et 30 p. 100 de l'effectif est susceptible de diminuer sensiblement le bénéfice escompté, du fait des saisies totales pour ladrerie généralisée ou de la nécessité d'assainir certaines carcasses par la congélation. Or le coût de cette opération est à Fort-Lamy de 13,77 F.CFA par kilogramme (GRABER, TROUETTE et CHAILLOUX).

En l'absence d'une méthode de diagnostic destinée à séparer les animaux parasités de ceux qui ne le sont pas, l'engraissement rapide des jeunes zébus en vue d'obtenir un «babybeef »n'est actuellement pas à recommander.

\section{CONCLUSIONS}

Une enquête effectuée dans la région de FortLamy et portant sur 458 zébus âgés de quelques jours à 10 ans et plus montre que la cysticercose à Cysticercus bovis est relativement peu répandue chez les veaux de lait (3 p. 100 environ).

Par contre, les possibilités d'infestation, de par le mode d'élevage traditionnel, deviennent favorables dès que les animaux commencent à être sevrés. Le plus grand nombre de jeunes porteurs de parasites est rencontré entre 6 mois et 2 ans (de 25 à 30 p. 100). Au-delà, le pourcentage diminue avec prédominance de cysticerques calcifiés ou en cours de calcification.

Il en résulte qu'en l'absence d'une méthode de diagnostic sérologique valable, l'achat des veaux de boucherie doit être effectué très tôt avant l'époque de sevrage et que l'élevage du «baby-beef » n'est pas à recommander, les risques de saisie pour cysticercose, avec ou sans assainissement, étant trop importants.

\section{SUMMARY}

\section{The bovine cysticercosis in the Fort-Lamy region.} The natural infestation of the young calves

In sucking calves slaughtered in the Fort-Lamy region, the cysticercosis by Cysticercus bovis is relatively not very frequent ( 3 p 100).

The infestation occurs from the weaning. Between one and two years, the number of infested animals reachs 31 p. 100.

The consequencies of those parasitic features for the fattening calves and the baby-beef are studied. 


\section{RESUMEN}

\section{La cisticercosis de los bovinos en la region de Fort-Lamy.} La infestación natural de los jovenes

En terneros de pecho sacrificados en la region de Fort-Lamy, la cisticercosis con Cysticerctis bovis es relativamente poco frecuente (3 p. 100).

Se produce la infestación a partir del destete. Entre 1 y 2 años de edad, el número de animales parasitados llega a 31 p. 100 y disminuye despues. Se consideran las consecuencias de dicha situación en lo concerniendo a los terneros de came y a los «baby-beef .

\section{BIBLIOGRAPHIE}

GRABER (M.), «La cysticercose bovine. Son importance dans les zones sahéliennes d’élevage de la République du Tchad». Rer. Elev. Méd. vét. Pays trop., 1959, 12, 2, 121-48.

GRABER (M.) et THOME (M.), « La cysticercose bovine en République du Tchad. Quelques réflexions sur la situation présente, l'étiologie, l'immunité et le traitement de cette zoonose", Rev. Elev. Méd. vét. Pays trop., 1964, 17, 3, 441-67.

GRABER (M.) et TABO (R.), « La cysticercoce bovine en milieu sédentaire et en milieu nomade $», R e v$. Elev. Méd. vét. Pays trop., 1968, 21, 1, 79-83.

GRABER (M.), TROUETTE (M.) et CHAILLOUX (A.), «Utilisation du froid pour la sté- rilisation des viandes ladres à l'abattoir de FortLamy », Bull. Inst. Int. Froid, 1970 (à paraître).

Mc INTOSH (A.) and MILLER (D.), "Bovine cysticercosis with special reference to the early developmental stages of $\mathrm{T}$. saginata 》, $A m$. J. vet. Res., 1960, 21, 81, 169-177.

Mc MANUS (D.), «Prenatal infection of calves with C. bovis $\%$, Vet. Res., 1960, 72, 41, 847-8.

SILVERMAN (P, H.) and HULLAND (T.J.), "Histological observations on bovine Cysticercosis », Res. Vet. Sc., 1961, 2, 3, 248-52.

URQUHART (G. M.), "Epizootiological and experimental studies on bovine Cysticercosis in East africa $\gg, J$. parasit. 1961, 47, 6, 857-69.

VAN GILS (J. H.), « Some data about Cysticercosis in the Netherlands ", Tijdschr. Diergeneesk., 1963, 88, 1488. 